Prospective Evaluation

\title{
A New Technique for the Treatment of Lumbar Facet Joint Syndrome Using Intra-articular Injection with Autologous Platelet Rich Plasma
}

Jiuping Wu, MSc, Zhenwu Du, MD, PhD, Yang Lv, MSc, Jun Zhang, MSc, Wei Xiong, MSc, Ruiqiang Wang, MSc, Rui Liu, MSc, Guizhen Zhang, MD, PhD, and Qinyi Liu, MD, PhD

From: Department Orthopedics, the Second Hospital of Jilin University,

Changchun, China

Address Correspondence: Qinyi Liu, MD, PhD Department of Orthopedics The Second Hospital of Jilin University

218 Ziqiang Street, Nanguan District Changchun, China E-mail: 1226527208@qq.com

Disclaimer: There was no external funding in the preparation of this manuscript. Conflict of interest: Each author certifies that he or she, or a member of his or her immediate

family, has no commercial association (i.e., consultancies, stock ownership, equity interest, patent/licensing arrangements, etc.) that might pose a conflict of interest in connection with the submitted manuscript.

Manuscript received: 01-03-2016 Revised manuscript received: 05-13-2016

Accepted for publication: o6-14-2016

Free full manuscript: www.painphysicianjournal.com
Background and Objectives: Lumbar facet joint syndrome is currently suggested to be a main source of axial low back pain, and a large portion of axial low back pain is caused by disorders in lumbar facet joints. Intra-articular injection is one of the most common treatment methods in the early clinical application. Therefore, we attempt to seek a new injectable material, autologous platelet rich plasma (PRP), to treat lumbar facet syndrome, as well as to assess its therapeutic effectiveness and safety.

Study Design: A prospective clinic evaluation.

Setting: The outpatient clinic of a single academic medical center.

Methods: Total 19 patients with lumbar facet joint syndrome (8 men, 11 women; mean ages: $52.53 \pm 6.79$ years, range: $38-62$ years) were enrolled to receive lumbar facet joint injection with autologous PRP under $x$-ray fluoroscopic control. Patients were followed up immediately, at one week, one month, 2 months, and 3 months following treatment, and the elements of this analysis included low back pain visual analogue scale (VAS) at rest and during flexion, Roland-Morris Disability Questionnaire (RMQ), Oswestry Disability Index (ODI), and modified MacNab criteria for the pain relief.

Results: All the 19 patients completed the intra-articular injections with autologous PRP successfully. At one week after treatment, low back pain reduced significantly compared with prior to treatment both at rest and during flexion. The outcomes were assessed as "good" or "excellent" for 9 patients (47.37\%) immediately after treatment, 14 patients $(73.68 \%)$ at one week, 15 patients (78.95\%) at one month, 15 patients $(78.95 \%)$ at 2 months, and 15 patients $(78.95 \%)$ at 3 months. Statistically significant differences were observed based on RMQ and a more than $10 \%$ improvement in lumbar functional capacity was observed based on ODI between pre-treatment and post-treatment. In addition, there were no severe relevant complications during the whole process of injection and follow-up period.

Limitations: A control group and the curative effect observations with longer follow-up may lead to a more convincing result for our study.

Conclusions: In the short-term period of 3 months, the new technique of lumbar facet joint injection with autologous PRP is effective and safe for patients with lumbar facet joint syndrome.

Key words: Low back pain, lumbar facet joint syndrome, autologous platelet rich plasma, intra-articular injection

Pain Physician 2016; 19:617-625 
ow back pain poses a threat to people's health and is the second most common reason for visiting a primary care physician (1). Some epidemiologic evidence suggests that the incidence of low back pain has been estimated to be $5 \%$ annually, but there is a significant increase in lifetime prevalence ranging from $60 \%$ to $90 \%(1-4)$. The causes of low back pain are complex, and mainly include lumbar intervertebral discs, facet joints, sacroiliac joints, exiting spinal nerve roots, ligaments, muscles, viscera, and other nonspinal reasons (4). Historically, lumbar disc herniation is considered to be the leading cause of low back pain. However, recent studies have shown that nearly $15 \%-$ $52 \%$ of patients have low back pain caused by lumbar facet joint syndrome $(3,5,6)$.

As the only synovial joints in the spine, facet joints are pretty much identical to other peripheral joints, consisting of a synovial capsule, synovial membrane, hyaline cartilage, and subchondral bone (7). Like many peripheral synovial joints, the facet joints may develop degenerative changes after overuse and injury. However, the exact reasons of lumbar facet joint syndrome are uncertain. Several studies have shown that there are multiple reasons for lumbar facet joint syndrome, such as capsular stretch, entrapment of synovial villi between the articular surfaces, nerve impingement by osteophytes, and release of inflammatory substances (8-12). Currently, attention has been focused on osteoarthritic changes that lead to lumbar facet joint syndrome. Some reports suggest that the radiographic features of facet joint osteoarthritis are osteoarthritis with narrowing, joint space narrowing, osteophytosis, joint hypertrophy, subchondral sclerosis, and bony deformity, which are similar to traditional peripheral osteoarthritis (13-16). Other reports reveal that multiple inflammatory cytokines such as necrosis factor- $\alpha$ (TNF $\alpha)$, interleukin-1 $\beta$ (IL-1 $\beta$ ), and interleukin-6 (IL-6), as well as inflammatory mediators such as prostaglandins are enriched in the facet joint tissues in degenerative lumbar facet joints $(10,17)$. Such situations also occur in other joints with osteoarthritis, such as limb joints $(18,19)$.

Various methods have been applied to the treatment of lumbar facet joint syndrome, including open denervation, percutaneous endoscopic denervation, radiofrequency denervation, kryodenervation, and local injection using different drugs (20), among which, radiofrequency denervation and intra-articular injections are 2 of the most commonly used methods. But the outcomes from these therapeutic methods are controversial. Hirsch et al (21) were the first to claim successful intra-articular injection of facet joints, since then, intraarticular injection has gradually become one of the vital therapeutic methods for lumbar facet joint syndrome. In 1976, Mooney and Robertson et al (22) used steroids and local anesthetics in intra-articular blocking. Subsequently, steroids, local anesthetics, and phenol seem to be beneficial for intra-articular injections of facet joints in many studies. However, the outcomes of intra-articular injection with different materials are controversial (10). Therefore, it is essential to find a new and effective injectable material for intra-articular injection.

Platelet rich plasma (PRP) consists of a high concentration of platelets derived from the patient's own peripheral venous blood. Current studies indicate that PRP is an appropriate injectable material with great potential in treating many different musculoskeletal disorders such as osteoarthritis, lateral epicondylitis, rotator cuff disease, Achilles and patella tendinopathy, hamstring injuries, and degenerative spine disease $(23,24)$. The positive effects of PRP are attributed to various growth factors and cytokines, including platelet-derived growth factor (PDGF), transforming growth factor- $\beta$ (TGF- $\beta$ ), fibroblast growth factor (FGF), insulinlike growth factor 1 (IGF-1), connective tissue growth factor (CTGF), epidermal growth factor (EGF), as well as bioactive proteins that influence the healing of tendons, ligaments, muscles, and bones $(25,26)$. These components play key roles in promoting cell proliferation, matrix regeneration, angiogenesis, and an anti-inflammatory effect (27-29). At present, a number of studies have proved the favorable efficacy of PRP in management of many musculoskeletal disorders. Whereas, to our knowledge, reports about intra-articular injection of PRP for the treatment of lumbar facet joint syndrome are rare.

In the present study, we applied PRP as a new material for intra-articular injections and evaluated its feasibility and safety in the treatment of lumbar facet joint syndrome.

\section{Methods}

\section{Ethics Approval}

Ethics approval was obtained from the ethics committee for lumbar facet joint injections using autologous PRP under x-ray fluoroscopic control. Patients signed an informed consent before the treatment.

\section{Patients}

A total of 19 patients with lumbar facet joint syn- 
drome ( 8 men, 11 women; mean ages: $52.53 \pm 6.79$ years, range: $38-62$ years) were enrolled in this study (Table 1). The diagnosis was based on clinical histories, clinical signs, physical examinations, imaging tests (mainly lumbosacral $x$-rays), and clinical experience of experts. The treated segments were mainly determined by lumbosacral $x$-rays, clinical signs, and clinical experience of experts.

The inclusive criteria were listed as follows:

- Continuous or intermittent low back pain;

- Local or paraspinal pain with or without radiation to the buttock, groin, or thigh;

- Increase of pain on flexion, rotation, or lateral bending, and with local excessive stress;

- Fracture like feelings when bending down;

- Experience of hard physical labor or sedentariness;

- Absence of neurological deficit;

- Lumbosacral $x$-rays showing findings of lumbar facet joint degenerative changes (osteoarthritis with narrowing, joint space narrowing, osteophytosis, joint hypertrophy, subchondral sclerosis, and bony deformity).

The exclusive criteria were listed as follows:

- Radicular neurologic complaints or with evident disc herniations;

- $\quad$ Prior surgery on the spine;

- Intolerance of local anesthesia and contrast medium.

Also, all the enrolled patients had a strong desire for treatment because of serious chronic low back pain, and a 3-month or longer follow-up after intervention was performed. What is more, all the patients were under enough physical discomfort to accept the intervention of lumbar facet joint injection.

\section{Preparation of Autologous PRP}

The PRP was prepared based on the standard 2-step centrifugation method. Under sterile conditions, approximately $5-10 \mathrm{~mL}$ of peripheral blood sample (depending on the number of treated levels) was collected with a sodium citrate coagulation test tube. Then the sample was first centrifuged at $200 \times \mathrm{g}$ for 10 minutes at room temperature to get whole serum supernatant and a small part of subnatant erythrocyte. Serum supernatant was subjected to the second centrifugation at $400 \times \mathrm{g}$ for 10 minutes to remove part of the platelet poor plasma. Finally, approximately $1-2 \mathrm{~mL}$ autologous PRP was collected in a clear syringe for injection as soon as possible. For every enrolled patients, the complete blood count $(C B C)$ in native peripheral blood before the

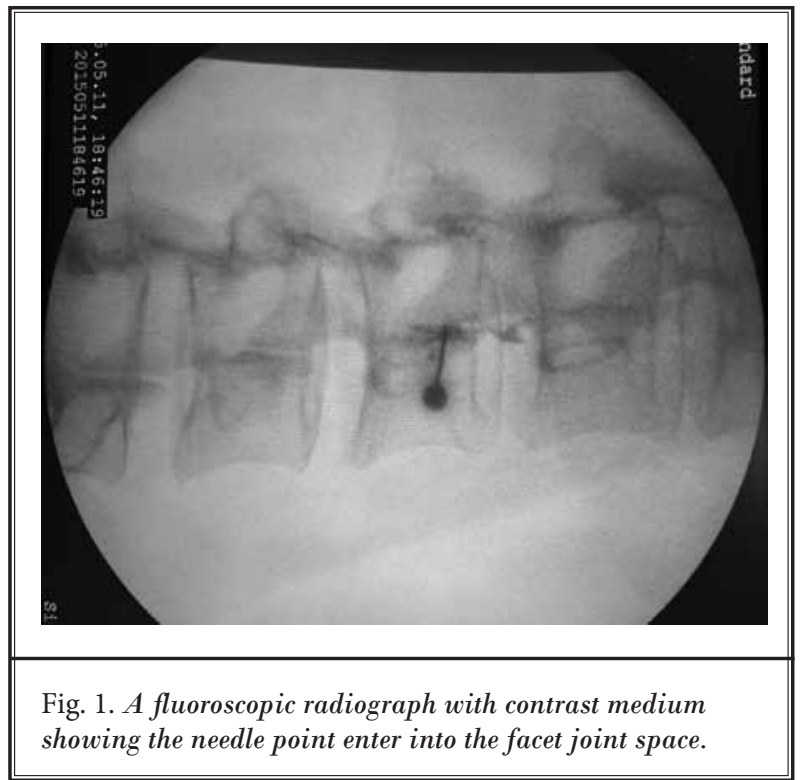

treatment and the platelet concentration in PRP after standard centrifugations were tested to ensure that the platelet concentration in the PRP was almost $4-5$ times greater than that in the native peripheral blood $(100-300 \times 109 / \mathrm{mL})$.

\section{Lumbar Facet Joint Injection}

Lumbar facet joint injection was performed by an experienced spine surgeon under fluoroscopy. The patients were placed prone on the operating table surrounded by a $\mathrm{C}$-arm, with a pillow under the abdomen to straighten the lumbar spine. The C-arm was rotated until the targeted lumbar facet joint space was clearly seen, when the beam of the $\mathrm{C}$-arm paralleled the open angle of the joint. The site for needle penetration was marked at this intersection of the beam of the C-arm and the skin. After the standard antisepsis of the skin was prepared, local anesthesia with $0.5 \%$ lidocaine was administered. A 21-G spinal needle was gently inserted into the facet joint space under fluoroscopic control. To verify the intra-articular positioning of the needle, $0.1-0.2 \mathrm{~mL}$ of nonionic contrast medium (Iohexol $15 \mathrm{~g} / 50 \mathrm{~mL}$ ) was injected (Fig. 1). The nonionic contrast medium was characterized by rapid metabolism, so there was little effect of the contrast medium injection on the PRP treatment. After successful intra-articular puncture, the targeted joint was injected with approximately $0.5 \mathrm{~mL}$ autologous PRP. The intra-articular injections were performed slowly with gentle pressure to avoid rupturing the joint capsule. After confirming that there was no obvious bleeding, the lumbar facet 
joint injection was successfully completed.

After the injection treatment, no puncture-related complications were observed within 4 hours. Also, patients were given information for pain relief in the hospital and were asked to rest as much as possible and avoid bend at the waist for one week after leaving the hospital. There was no anti-inflammatory treatment for patients during the follow-up period.

\section{Follow-up and Clinical Evaluation}

All the patients were followed up for 3 months. The interviews were mostly conducted by telephone according to the following criteria and scales: low back pain visual analogue scale (VAS) at rest and during flexion, Roland-Morris Disability Questionnaire (RMQ), Oswestry Disability Index (ODI), modified MacNab criteria for the relief of pain (excellent, good, fair, or poor). VAS is a subjective numerical pain rating scale ranging from 0 to 10 , where 0 indicates no pain and 10 indicates the worst pain ever experienced. RMQ was designed to assess back pain as experienced by the patient in the last 24 hours, and the outcome was a total score, a score of 0 represented no disability and a score of 24 represented the maximum disability. ODI is currently considered as the gold standard for measuring degree of disability and estimating quality of life in a patient with low back pain. The ODI is comprised of 10 items which reflect the patient's ability to manage their everyday life while dealing with their pain. Each item in the ODI had 6 options to represent a score from 0 to 5 . A percentage score showed: total score of the patient / total score possible $\times 100 \%$. A $10 \%$ change has been identified as being clinically meaningful. The result had 5 levels: minimal disability $(0 \%-20 \%)$, moderate disability $(21 \%-40 \%)$, severe disability $(41 \%-60 \%)$, crippled $(61 \%-80 \%)$, and bed-bound or exaggerating symptoms $(81 \%-100 \%)$.

All the interviews were conducted for patients before treatment and after treatment immediately, at one week, one month, 2 months, and 3 months, respectively. Next, the collection and the statistical analysis of clinical data were performed to evaluate the effectiveness of this therapeutic method.

\section{Statistical Analysis}

The SPSS version 19.0 program was used for data analysis. All the data were expressed as means \pm standard deviation (SD). The paired t-test was used for comparing the difference in VAS, RMQ, and ODI before and after treatment. The $95 \%$ confidence intervals were determined and a $P$ value of less than 0.05 was considered statistically significant. All the graphs were constructed by using GraphPad Prism 5.0.

\section{Results}

All 19 patients received the initial 3-month followup after a successful lumbar facet joint injection, and no patient was lost in the follow-up period. Based on our statistics, each patient had more than one segment to be treated. Among all the cases, 12 patients were injected on one side and 7 patients on both sides. The history of pain for patients lasted continuously or intermittently, ranging from 2 months to 16 years. Most patients had a history of taking pain medications and presented with slight pain radiation to other areas. Other basic characteristics of patients are listed in Table 1.

During the follow-up, every enrolled patient completed the questionnaires with VAS scores for low back pain and pain VAS scores during flexion. The outcomes showed that the low back pain was relieved in patients treated with PRP intra-articular injections (Fig. 2). The mean VAS scores at rest were 7.05 before treatment, 6.68, $4.89,3.21,3.37$, and 2.63 immediately, at one week, one month, 2 months, and 3 months after treatment. The scores weres 8.42, 8.05, 6.05, 4.21, 3.89, and 2.95 during flexion, respectively. Compared with the scores prior to treatment, the pain at rest and during flexion were significantly reduced at one week, one month, 2 months, and 3 months after treatment $(P<0.05)$. However, there were no significant differences of scores between immediately after treatment and prior to treatment $(P>0.05)$.

As shown in Fig. 3, RMQ scores were significantly reduced after lumbar facet joint injections. The mean scores of RMQ were reduced gradually in a time-dependent manner after treatment. Moreover, there was significant difference in the RMQ scores between pre-treatment and post-treatment $(P<0.05)$.

After lumbar facet joint injections with autologous PRP, the mean scores of ODI were decreased significantly compared with that prior to treatment (no less than $10 \%$ change occurred at any follow-up visit compared with pre-treatment). Prior to treatment, 17 patients $(89.47 \%)$ were reported to be at the severe disability level or above, only 2 patients (10.53\%) had moderate disability. However, at 3 months after treatment, all the 19 patients $(100 \%)$ were reported to be at the moderate disability level or below (Table 2).

According to the modified MacNab criteria, 9 (43.37\%), 14 (73.68\%), $15(78.95 \%), 15$ (78.95\%), and $15(78.95 \%)$ patients were considered as "excellent" or 
Autologous PRP Injection for Lumbar Facet Joint Syndrome

Table 1. The basic characteristics of the enrolled patients with low back pain.

\begin{tabular}{|c|c|c|c|c|c|c|c|}
\hline \multirow[b]{2}{*}{ No. } & \multirow{2}{*}{$\begin{array}{c}\text { Age } \\
\text { (years) }\end{array}$} & \multirow[b]{2}{*}{ Gender } & \multicolumn{5}{|c|}{ L Low back pain characteristics } \\
\hline & & & $\begin{array}{c}\text { Sides of } \\
\text { pain }\end{array}$ & $\begin{array}{c}\text { History of } \\
\text { pain }\end{array}$ & Radiation to other areas & $\begin{array}{c}\text { Pain } \\
\text { medications }\end{array}$ & Treated segments \\
\hline 1 & 46 & Female & Bilateral & 3 years & Bilateral buttocks & No & L1/L2, L2/L3 Bilateral \\
\hline 2 & 38 & Male & Left & 2 months & No & Yes & L2/L3, L3/L4 Left \\
\hline 3 & 42 & Male & Right & 10 years & Right mid-thigh & Yes & L2/L3, L3/L4, L4/L5 Right \\
\hline 4 & 56 & Female & Left & 4 years & Left mid-thigh & Yes & L2/L3, L3/L4, L4/L5 Left \\
\hline 5 & 53 & Female & Left & 16 years & Left buttock and groin & Yes & L3/L4, L4/L5 Left \\
\hline 6 & 51 & Female & Bilateral & 5 years & Bilateral groins & Yes & L3/L4, L4/L5 Bilateral \\
\hline 7 & 46 & Female & Left & 2 years & Left mid-thigh & No & L3/L4, L4/L5, L5/S1 Left \\
\hline 8 & 61 & Female & Bilateral & 2 years & Bilateral groins & No & L3/L4, L4/L5 Bilateral \\
\hline 9 & 51 & Male & Right & 4 years & Right mid-thigh & Yes & L3/L4, L4/L5 Right \\
\hline 10 & 42 & Female & Bilateral & 1 year & No & Yes & L2/L3, L3/L4 Bilateral \\
\hline 11 & 52 & Female & Right & 5 years & $\begin{array}{l}\text { Bilateral groins and upper } \\
\text { thighs }\end{array}$ & Yes & L2/L3, L3/L4, L4/L5 Right \\
\hline 12 & 46 & Male & Bilateral & 2 years & Bilateral groins & No & L3/L4, L5/S1 Bilateral \\
\hline 13 & 57 & Male & Right & 1 year & Right buttock & No & L2/L3, L3/L4, L4/L5 Right \\
\hline 14 & 53 & Female & Bilateral & 3 years & Bilateral buttocks and groins & Yes & L2/L3, L3/L4 Bilateral \\
\hline 15 & 62 & Female & Left & 5 years & Left mid-thigh & Yes & L3/L4, L4/L5, L5/S1 Left \\
\hline 16 & 43 & Male & Right & 4 months & No & Yes & L2/L3, L3/L4 Right \\
\hline 17 & 59 & Female & Right & 6 years & Right mid-thigh & Yes & L3/L4, L4/L5, L5/S1 Right \\
\hline 18 & 49 & Male & Left & 1 year & No & Yes & L2/L3, L3/L4, L4/L5 Left \\
\hline 19 & 53 & Male & Bilateral & 3 years & Bilateral buttocks & Yes & L4/L5, L5/S1 Bilateral \\
\hline
\end{tabular}

"good" immediately, at one week, one month, 2 months, and 3 months after treatment with autologous PRP injection, respectively. Only one patient had reported a poor outcome at one week after treatment. The detailed results of the modified MacNab criteria are shown in Fig. 4.

There were no cases of infection, rejection reaction, nerve injury or injection-related complications during the 3-month follow-up period. Immediately after treatment, low back pain was aggravated in four patients, but there were no nerve injuries in these patients after general and elemental neurological exams. Thus, lumbar facet joint injection with autologous PRP was sufficiently safe for patients with lumbar facet joint syndrome.

\section{Discussion}

Lumbar facet joint syndrome is defined as a kind of common low back pain deriving from the lumbar facet joints, which has a strong impact on activities of daily living. As the only

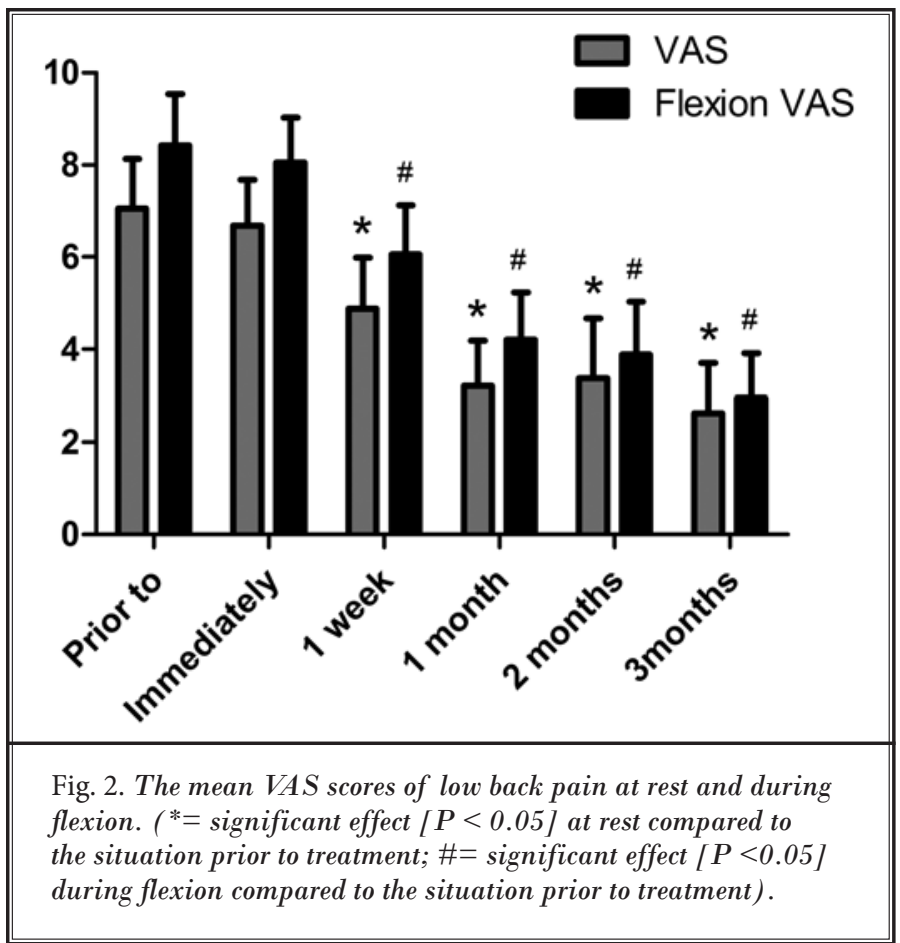


synovial joints in the spine, facet joints and peripheral synovial joints have extremely similar compositions. In addition, the degenerative changes of facet joints are equivalent to other peripheral joints (7). At present, different studies have described multiple therapeutic techniques to manage lumbar facet joint syndrome, and intra-articular injection is one of the most important methods $(2,20,30)$. Injection therapy is common for lumbar facet joint syndrome and has been modified with multiple drugs. However, a previous study suggests that the outcomes of intra-articular injection with different drugs are controversial and may result in different levels of drug-related complications (20).

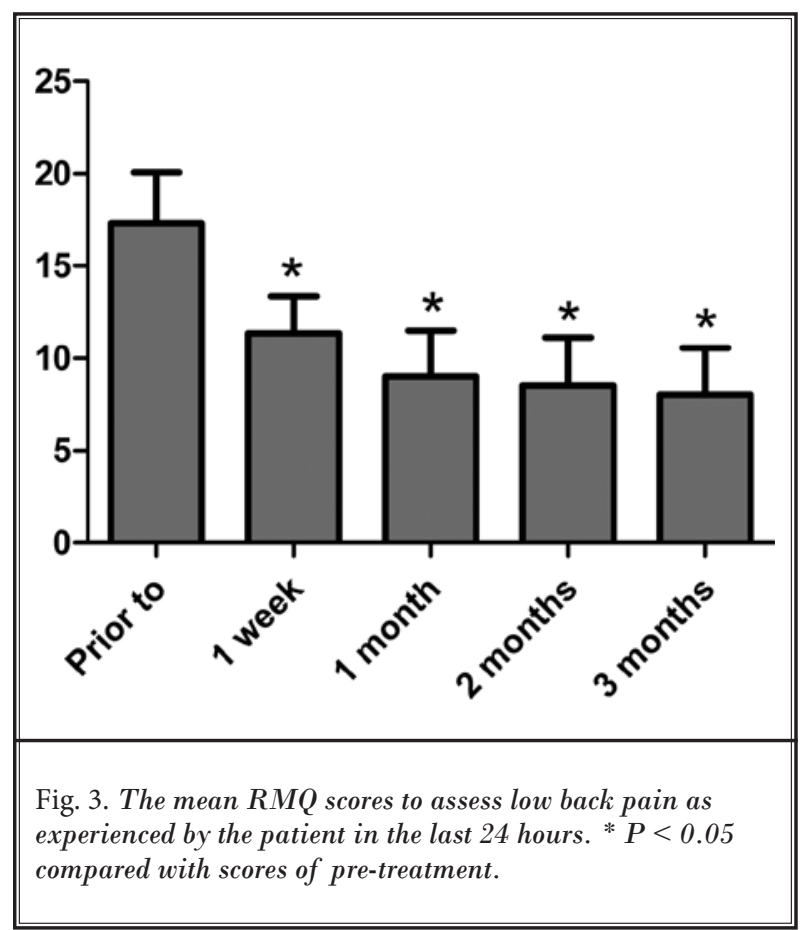

Therefore, it is critical to seek new injectable materials to be used for intra-articular facet joint injection for the treatment of lumbar facet joint syndrome.

Recently, PRP therapy as a safe, nonsurgical, biological treatment for osteoarthritis and musculoskeletal repair has gained a lot of attention. Since PRP is prepared from autologous blood, theoretically there are minimal risks for toxicity and side effects (31). Due to these features, PRP becomes a very appropriate material for intra-articular injection. However, to the best of our knowledge, there are no related studies about using autologous PRP to treat lumbar facet joint syndrome. PRP therapy is a new technique for the treatment of lumbar facet joint syndrome. Previous studies have indicated that reparative efficacy with PRP can be expected with 4 - 5 times of normal blood level, whereas no further enhancement is observed for PRP with much higher platelet concentrations (32). According to the results of our preliminary experiment, the platelet-enriched plasma with $4-5$ times the platelet concentration may get a more positive outcome for lumbar facet joint syndrome.

The results of our study demonstrated that facet joint injection using autologous PRP was an effective therapeutic method. Based on the modified MacNab criteria, $78.95 \%$ of the patients were satisfied with the treatment results at 3 months after PRP injections. Compared pain levels before treatment, the level of low back pain after treatment was significantly decreased. In regard to lumbar disability, the results of RMQ and ODI showed that the degree of lumbar disability was obviously reduced and the quality of life had an anticipated improvement. However, we did not have a control group in this study, the objective evaluations of the results might have a limitation.

Mooney and Robertson et al (22) first declared that intra-articular facet joint injection with steroids and lo-

Table 2. Overall results of ODI prior to treatment and at one week, one month, 2 months, and 3 months after the treatment.

\begin{tabular}{|l|c|c|c|c|c||}
\hline \multirow{2}{*}{} & \multirow{2}{*}{ Prior to } & \multicolumn{4}{|c||}{ After } \\
\cline { 3 - 6 } & & 1 week & 1 month & 2 months & 3 months \\
\hline Means \pm SDs & $54.32 \pm 13.94 \%$ & $39.47 \pm 7.77 \%$ & $27.79 \pm 6.63 \%$ & $24.63 \pm 8.19 \%$ & $26.32 \pm 5.67 \%$ \\
\hline Minimal disability & $0(0 \%)$ & $0(0 \%)$ & $4(21.05 \%)$ & $8(42.11 \%)$ & $4(21.05 \%)$ \\
\hline Moderate disability & $2(10.53 \%)$ & $12(63.16 \%)$ & $15(78.95 \%)$ & $10(52.63 \%)$ & $15(78.95 \%)$ \\
\hline Severe disability & $10(52.63 \%)$ & $7(36.84 \%)$ & $0(0 \%)$ & $1(5.26 \%)$ & $0(0 \%)$ \\
\hline Crippled & $6(31.58 \%)$ & $0(0 \%)$ & $0(0 \%)$ & $0(0 \%)$ & $0(0 \%)$ \\
\hline $\begin{array}{l}\text { Bed-bound or exaggerating } \\
\text { symptoms }\end{array}$ & $1(5.26 \%)$ & $0(0 \%)$ & $0(0 \%)$ & $0(0 \%)$ & $0(0 \%)$ \\
\hline
\end{tabular}




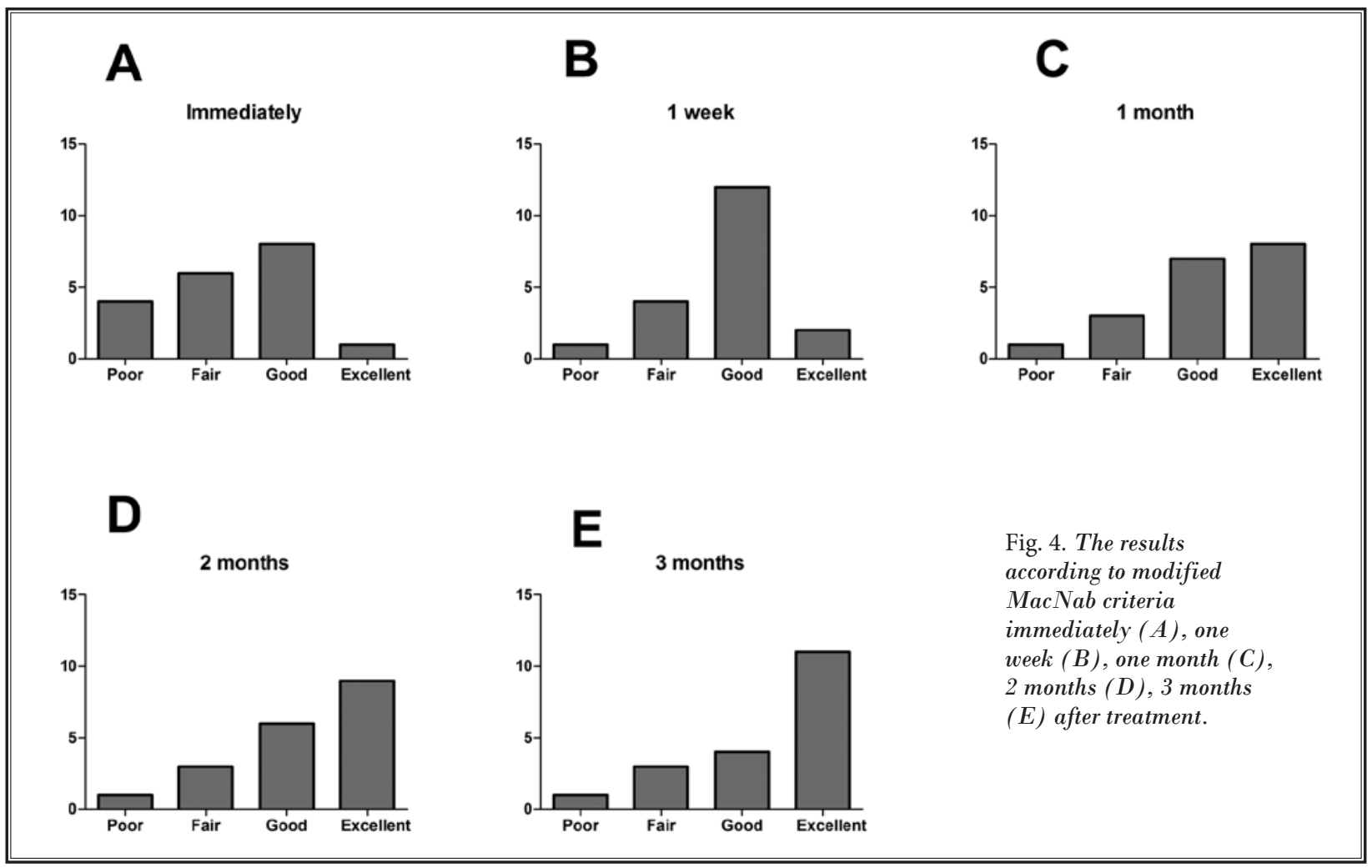

cal anesthetics got a satisfying outcome with $32 \%$ of patients experiencing "complete relief" in a 6-month follow-up. Since then, injection therapy of facet joints has become a routine treatment option for lumbar facet joint syndrome, and steroids combined with local anesthetics have become the most used injectable materials. A systematic review has concluded that the low back pain relief after intra-articular steroid injection ranges from $18 \%$ to $63 \%$ (33). Pneumaticos et al (34) reported that $87 \%$ of patients had an improvement in the pain score at one month after steroid and local anesthetic injections. Schulte et al (20) found that, according to the MacNab criteria, $41 \%$ (16 patients) of patients with lumbar facet joint syndrome gained excellent or good outcomes at 3 months after intra-articular injections using a standardized protocol (prednisolone acetate, lidocaine $1 \%$, phenol $5 \%$ ). Ribeiro et al (35) showed that the VAS score was reduced from 7.0 before the intervention to 4.7 at 3 months $(P<0.001)$ and $51.9 \%$ of patients expressed satisfied outcomes with significant pain relief at 3 months after facet joint injections with $1 \mathrm{~mL}$ of lidocaine and $1 \mathrm{~mL}$ of triamcinolone hexacetonide. Our data showed that, based on the modified MacNab criteria, $78.95 \%$ of the patients were assessed to have excellent or good outcomes at 3 months after PRP injections, which suggested that application of PRP might be more effective than the standardized protocol. Nonetheless, in many systematic reviews, investigators take a skeptical attitude about the efficacy of steroid facet joint injections $(36,37)$. In a randomized and controlled study, Carette et al (38) reported that injecting steroid into the facet joints showed little effect on the treatment of patients with chronic low back pain. The mean pain VAS score for patients with steroid injection at one month was 4.7, which was similar with that at 3 month (38). According to our clinical experience, intra-articular facet joint injections with steroid and (or) local anesthetic may show significant pain relief in the short-term, but the long-term therapeutic effects are uncertain. Our results revealed that the mean VAS scores at rest and during flexion were 2.63 and 2.95, respectively, at the 3 month evaluation after PRP injection, which were decreased compared with that at one month (3.21 and 4.21). All these findings suggested that PRP injection showed more effect on low back pain than injection with steroids, especially in a longer term period. In addition, our treatment method could significantly improve the quality of life in a short-term 
of 3 months. However, the lack of systematic observation of PRP therapy for a longer follow-up period is a limitation in our study.

In the study of provocative, historical, and other tests for lumbar facet joint pain, Jackson et al (39) demonstrated that independent variables affecting pain relief after intra-articular facet joint injection were older age, prior history of low back pain, normal gait, maximum pain on extension following forward flexion in the standing position, and the absence of leg pain, muscle spasm, and aggravation of pain on Valsava. However, none of these variables are unique to the patients responding to lumbar facet joint injection. The accurate diagnosis of lumbar facet joint syndrome is controversial, and it depends more on an intricate comprehensive diagnostic method including clinical factors, radiologic findings, and invasive diagnostic blocks. Currently, many surgeons and experts believe that diagnostic blocks are the most reliable means for diagnosing lumbar facet joint as a generator of low back pain $(4,40,41)$. However, in order to eliminate the interferences from diagnostic blocks on the evaluation of low back pain from diagnostic blocks, we preferred to diagnose lumbar facet joint syndrome using clinical histories, clinical signs, physical examinations, imaging tests (mainly lumbosacral x-rays), and the clinical experience of experts.

Schulte et al (20) noted that short-lasting self-limiting mild side effects (increased back pain, numbness, heartburn, headache, allergy) were found in $26 \%$ of patients. Other nonsignificant, infrequent adverse events related to injections are also reported in previous clinical studies $(6,38,42,43)$. What's more, although previous studies indicated that the frequency of adverse systemic events was low after the use of systemic steroids at high does, most patients were still concerned about the potential health problems derived from using too much steroid. However, the autologous PRP, which is derived from autologous blood, can completely relieve this concern. According to our data, no severe complications were observed after intra-articular facet joint injections with autologous PRP. The increased low back pain from 4 patients may be due to sight tissue damage from the puncture and expansion of the joint capsule. Therefore, it is safe for patients with lumbar facet joint syndrome with PRP.

Due to the lack of clinical studies on the application of autologous PRP for treating patients with lumbar facet joint syndrome, there are still some limitations in our study. A control group and the curative effect observations with more than 3 months follow-up may lead to a more convincing result.

\section{Conclusion}

Autologous PRP is a promising new option for the treatment of patients with lumbar facet joint syndrome. Our study demonstrates that this new treatment technique with autologous PRP injection is safe and effective for the patients with lumbar facet joint syndrome and may have vast application going forward. Further studies with larger numbers of patients and longer follow-up periods are needed to validate our findings.

\section{References}

1. Slipman CW, Bhat AL, Gilchrist RV, Issac Z, Chou L, Lenrow DA. A critical review of the evidence for the use of zygapophysial injections and radiofrequency denervation in the treatment of low back pain. The Spine Journal 2003; 3:310-316.

2. Civelek E, Cansever T, Kabatas S, Kircelli A, Yilmaz C, Musluman M, Ofluoglu D, Caner $\mathrm{H}$. Comparison of effectiveness of facet joint injection and radiofrequency denervation in chronic low back pain. Turk Neurosurg 2012; 22:200-206.

3. Proietti L, Schiro GR, Sessa S, Scaramuzzo $L$. The impact of sagittal balance on low back pain in patients treated with zygoapophysial facet joint injection. Eur Spine J 2014; 23:628-633.

4. Dreyer SJ, Dreyfuss PH. Low back pain and the zygapophysial (facet) joints. Arch
Phys Med Rehabil 1996; 77:290-300.

5. Staender M, Maerz U, Tonn JC, Steude U. Computerized tomography-guided kryorhizotomy in 76 patients with lumbar facet joint syndrome. J Neurosurg Spine 2005; 3:444-449.

6. Lilius G, Laasonen EM, Myllynen P, Harilainen A, Gronlund G. Lumbar facet joint syndrome. A randomised clinical trial. J Bone Joint Surg Br 1989; 71:681-684.

7. Maataoui A, VogI TJ, Khan MF. Magnetic resonance imaging-based interpretation of degenerative changes in the lower lumbar segments and therapeutic consequences. World J Radiol 2015; 7:194-197.

8. Tessitore E, Molliqaj G, Schatlo B, Schaller K. Clinical evaluation and surgical decision making for patients with lumbar discogenic pain and facet syn- drome. Eur J Radiol 2015; 84:765-770.

9. Gellhorn AC, Katz JN, Suri P. Osteoarthritis of the spine: The facet joints. Nat Rev Rheumatol 2013; 9:216-224.

10. Igarashi A, Kikuchi S, Konno S, Olmarker K. Inflammatory cytokines released from the facet joint tissue in degenerative lumbar spinal disorders. Spine (Phila Pa 1976) 2004; 29:2091-2095.

11. Schulte TL, Filler TJ, Struwe P, Liem D, Bullmann V. Intra-articular meniscoid folds in thoracic zygapophysial joints. Spine (Phila Pa 1976) 2010; 35:E191-E197.

12. Kim JS, Kroin JS, Buvanendran A, Li X, van Wijnen AJ, Tuman KJ, Im HJ. Characterization of a new animal model for evaluation and treatment of back pain due to lumbar facet joint osteoarthritis. 
Arthritis Rheum 2011; 63:2966-2973.

13. Carrera GF, Williams AL. Current concepts in evaluation of the lumbar facet joints. Crit Rev Diagn Imaging 1984; 21:85-104.

14. Schwarzer AC, Wang SC, O'Driscoll D, Harrington T, Bogduk N, Laurent R. The ability of computed tomography to identify a painful zygapophysial joint in patients with chronic low back pain. Spine (Phila Pa 1976) 1995; 20:907-912.

15. Cohen SP, Hurley RW, Christo PJ, Winkley J, Mohiuddin MM, Stojanovic MP. Clinical predictors of success and failure for lumbar facet radiofrequency denervation. Clin J Pain 2007; 23:45-52.

16. Stojanovic MP, Sethee J, Mohiuddin M, Cheng J, Barker A, Wang J, Palmer W, Huang A, Cohen SP. MRI analysis of the lumbar spine: $C$ an it predict response to diagnostic and therapeutic facet procedures? Clin J Pain 2010; 26:110-115.

17. Willburger RE, Wittenberg RH. Prostaglandin release from lumbar disc and facet joint tissue. Spine (Phila Pa 1976) 1994; 19:2068-2070.

18. Pelletier JP, Martel-Pelletier J, Abramson SB. Osteoarthritis, an inflammatory disease: Potential implication for the selection of new therapeutic targets. Arthritis Rheum 2001; 44:1237-1247.

19. Smith MD, Triantafillou S, Parker A Youssef PP, Coleman M. Synovial membrane inflammation and cytokine production in patients with early osteoarthritis. J Rheumatol 1997; 24:365-371.

20. Schulte TL, Pietila TA, Heidenreich J, Brock M, Stendel R. Injection therapy of lumbar facet syndrome: A prospective study. Acta Neurochir (Wien) 2006; 148:1165-1172; discussion 1172.

21. Hirsch C, Ingelmark BE, Miller M. The anatomical basis for low back pain. Studies on the presence of sensory nerve endings in ligamentous, capsular and intervertebral disc structures in the human lumbar spine. Acta Orthop Scand 1963; 33:1-17.

22. Mooney V, Robertson J. The facet syndrome. Clin Orthop Relat Res 1976; 115:149-156.

23. Cook JL, Smith PA, Bozynski CC, Kuroki K, Cook CR, Stoker AM, Pfeiffer FM. Multiple injections of leukore- duced platelet rich plasma reduce pain and functional impairment in a canine model of ACL and meniscal deficiency. J Orthop Res 2015; 32:495-505.

24. Meheux CJ, McCulloch PC, Lintner DM, Varner KE, Harris JD. Efficacy of intraarticular platelet-rich plasma injections in knee osteoarthritis: A systematic review. Arthroscopy 2015; 34:607-615.

25. Kabiri A, Esfandiari E, Esmaeili A, Hashemibeni B, Pourazar A, Mardani M. Platelet-rich plasma application in chondrogenesis. Adv Biomed Res 2014; 3:138.

26. Civinini R, Nistri L, Martini C, Redl B, Ristori G, Innocenti M. Growth factors in the treatment of early osteoarthritis. Clin Cases Miner Bone Metab 2013; 10:26-29.

27. Bendinelli P, Matteucci E, Dogliotti G, Corsi MM, Banfi G, Maroni P, Desiderio MA. Molecular basis of anti-inflammatory action of platelet-rich plasma on human chondrocytes: Mechanisms of NF-kappaB inhibition via HGF. J Cell Physiol 2010; 225:757-766.

28. Mazzocca AD, McCarthy MB, Intravia J, Beitzel K, Apostolakos J, Cote MP, Bradley J, Arciero RA. An in vitro evaluation of the anti-inflammatory effects of platelet-rich plasma, ketorolac, and methylprednisolone. Arthroscopy 2013; 29:675-683.

29. Zhang J, Middleton KK, Fu FH, Im HJ, Wang JH. HGF mediates the anti-inflammatory effects of PRP on injured tendons. PLoS One 2013; 8:e67303.

30. Lakemeier S, Lind M, Schultz W, FuchsWinkelmann S, Timmesfeld N, Foelsch C, Peterlein CD. A comparison of intraarticular lumbar facet joint steroid injections and lumbar facet joint radiofrequency denervation in the treatment of low back pain: A randomized, controlled, double-blind trial. Anesth Analg 2013; 117:228-235.

31. Everts PA, Knape JT, Weibrich G, Schonberger JP, Hoffmann J, Overdevest EP, Box HA, van Zundert A. Platelet-rich plasma and platelet gel: A review. J Extra Corpor Technol 2006; 38:174-187.

32. Freymiller EG. Platelet-rich plasma: evidence to support its use. J Oral Maxillofac Surg 2004; 62:1046; author reply 1047-1048.
33. Cohen SP, Raja SN. Pathogenesis, diagnosis, and treatment of lumbar zygapophysial (facet) joint pain. Anesthesiology 2007; 106:591-614.

34. Pneumaticos SG, Chatziioannou SN, Hipp JA, Moore WH, Esses SI. Low back pain: Prediction of short-term outcome of facet joint injection with bone scintigraphy. Radiology 2006; 238:693-698.

35. Ribeiro LH, Furtado RN, Konai MS, Andreo AB, Rosenfeld A, Natour J. Effect of facet joint injection versus systemic steroids in low back pain: A randomized controlled trial. Spine (Phila Pa 1976) 2013; 38:1995-2002.

36. Datta S, Lee M, Falco FJ, Bryce DA, Hayek SM. Systematic assessment of diagnostic accuracy and therapeutic utility of lumbar facet joint interventions. Pain Physician 2009; 12:437-460.

37. Bogduk N. Evidence-informed management of chronic low back pain with facet injections and radiofrequency neurotomy. Spine J 2008; 8:56-64.

38. Carette $S$, Marcoux S, Truchon R, Grondin C, Gagnon J, Allard Y, Latulippe M. A controlled trial of corticosteroid injections into facet joints for chronic low back pain. N Engl J Med 1991; 325:1002-1007.

39. Jackson RP, Jacobs RR, Montesano PX. 1988 Volvo award in clinical sciences. Facet joint injection in low-back pain. A prospective statistical study. Spine 1988;13:966-971.

40. Sowa G. Facet-mediated pain. Dis Mon 2005; 51:18-33.

41. Bogduk N. International Spinal Injection Society guidelines for the performance of spinal injection procedures. Part 1: Zygapophysial joint blocks. Clin J Pain 1997; 13:285-302.

42. Manchikanti L, Pampati V, Bakhit CE, Rivera JJ, Beyer CD, Damron KS, Barnhill RC. Effectiveness of lumbar facet joint nerve blocks in chronic low back pain: A randomized clinical trial. Pain Physician 2001; 4:101-117.

43. Fuchs S, Erbe T, Fischer HL, Tibesku CO. Intraarticular hyaluronic acid versus glucocorticoid injections for nonradicular pain in the lumbar spine.] Vasc Interv Radiol 2005; 16:1493-1498. 
